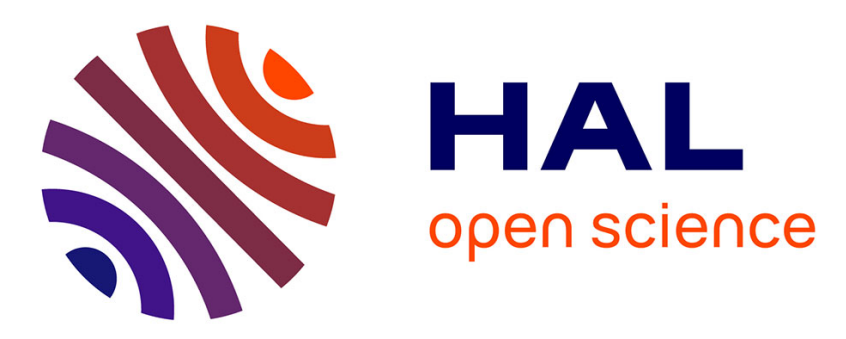

\title{
Charge Transport Along Phenylenevinylene Molecular Wires
}

Laurens Siebbeles, Paulette Prins, Ferdinand Grozema

\section{To cite this version:}

Laurens Siebbeles, Paulette Prins, Ferdinand Grozema. Charge Transport Along Phenylenevinylene Molecular Wires. Molecular Simulation, 2006, 32 (09), pp.695-705. 10.1080/08927020600835657. hal-00514986

\section{HAL Id: hal-00514986 \\ https://hal.science/hal-00514986}

Submitted on 4 Sep 2010

HAL is a multi-disciplinary open access archive for the deposit and dissemination of scientific research documents, whether they are published or not. The documents may come from teaching and research institutions in France or abroad, or from public or private research centers.
L'archive ouverte pluridisciplinaire HAL, est destinée au dépôt et à la diffusion de documents scientifiques de niveau recherche, publiés ou non, émanant des établissements d'enseignement et de recherche français ou étrangers, des laboratoires publics ou privés. 


\section{Molecular Simulation}

Journal of

Experimental Nanoscience

- Taylor \& Francis

Taglor \& Francis Group

\section{Charge Transport Along Phenylenevinylene Molecular Wires}

\begin{tabular}{|c|c|}
\hline Journal: & Molecular Simulation/Journal of Experimental Nanoscience \\
\hline Manuscript ID: & GMOS-2006-0036.R1 \\
\hline Journal: & Molecular Simulation \\
\hline $\begin{array}{r}\text { Date Submitted by the } \\
\text { Author: }\end{array}$ & 01-May-2006 \\
\hline Complete List of Authors: & $\begin{array}{l}\text { Siebbeles, Laurens; TU Delft, DelftChemTech } \\
\text { Prins, Paulette; TU Delft, DelftChemTech } \\
\text { Grozema, Ferdinand; TU Delft, DelftChemTech }\end{array}$ \\
\hline Keywords: & charge mobility, molecular wire, conjugated polymer \\
\hline
\end{tabular}

SCHOLARONE $^{m}$
Manuscripts 


\section{Page 1 of 43}
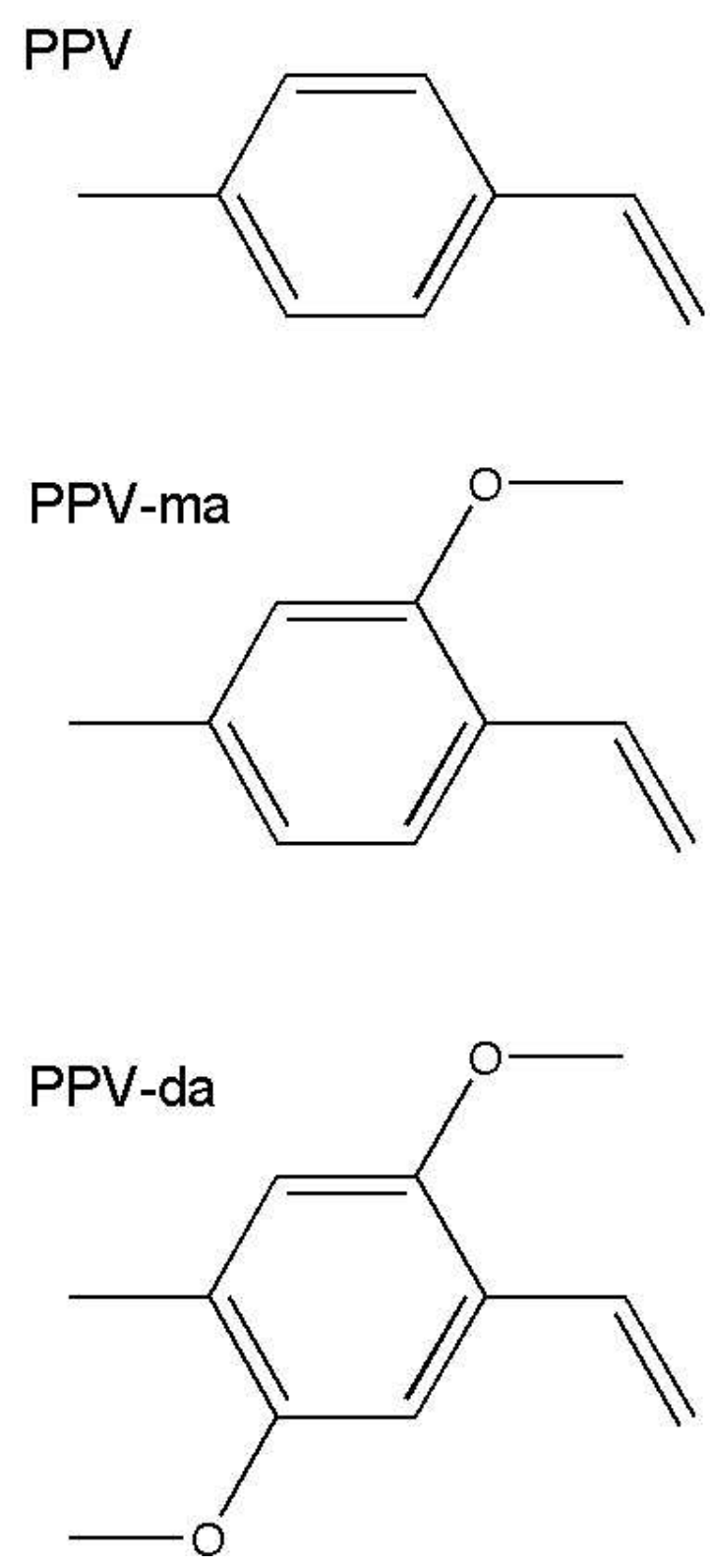

$31 \times 70 \mathrm{~mm}(300 \times 300$ DPI $)$ 


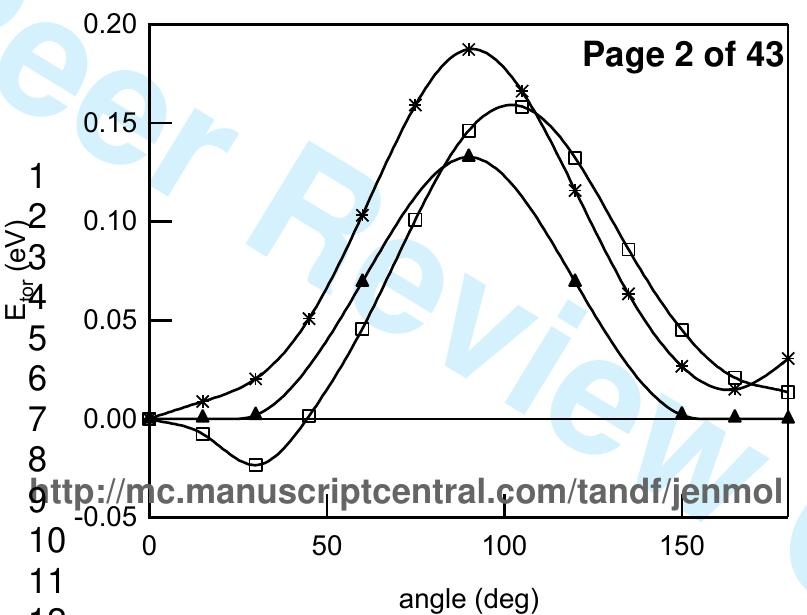




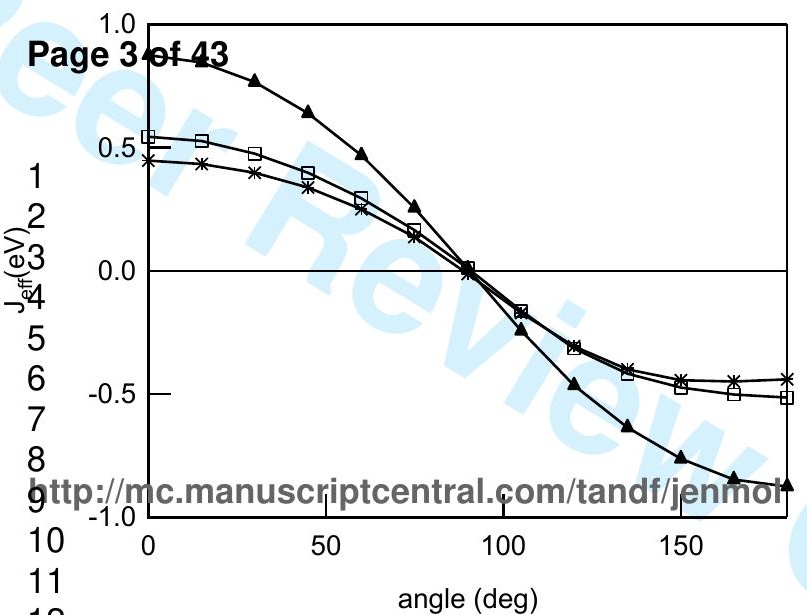




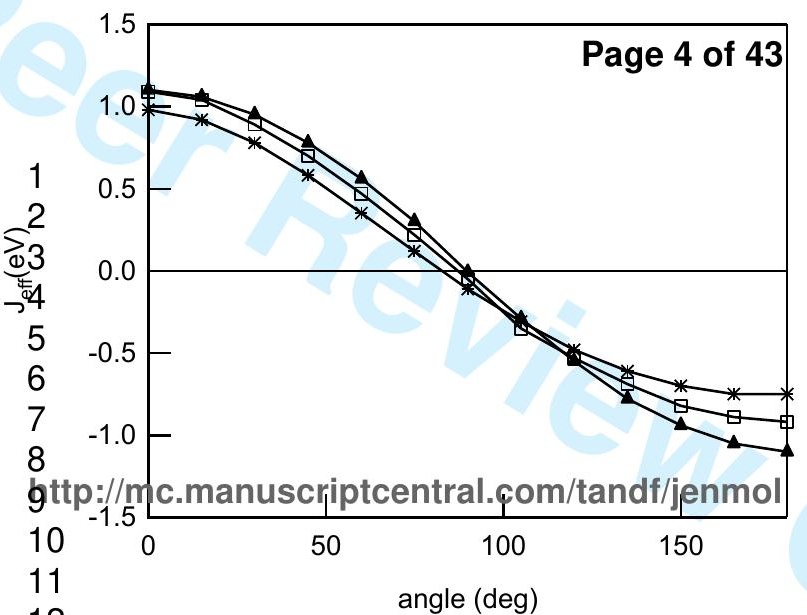




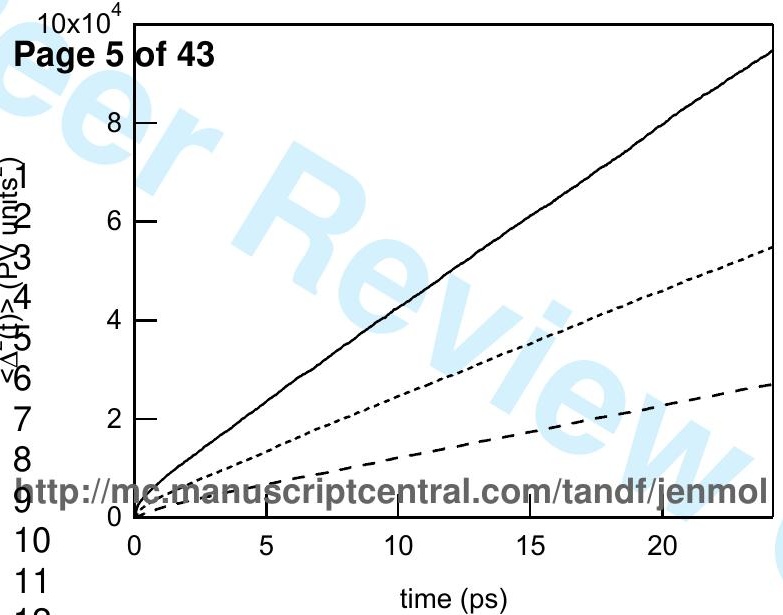




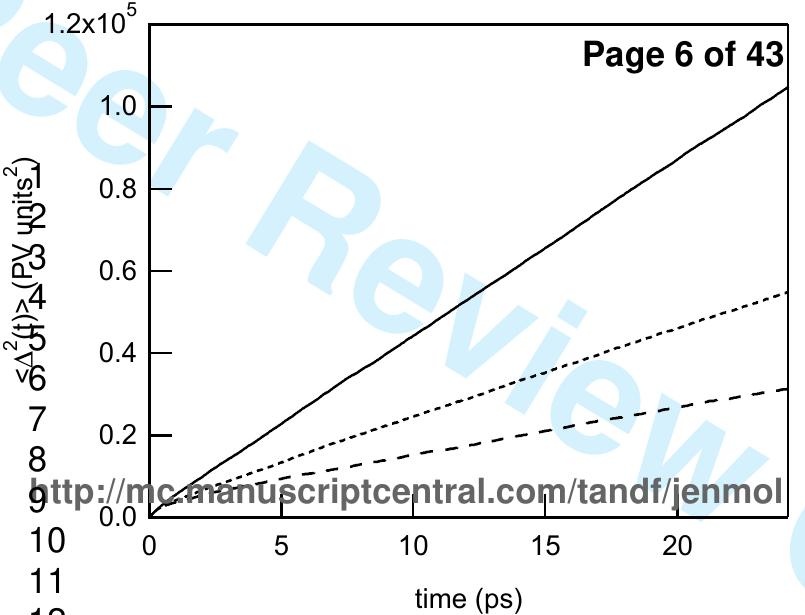




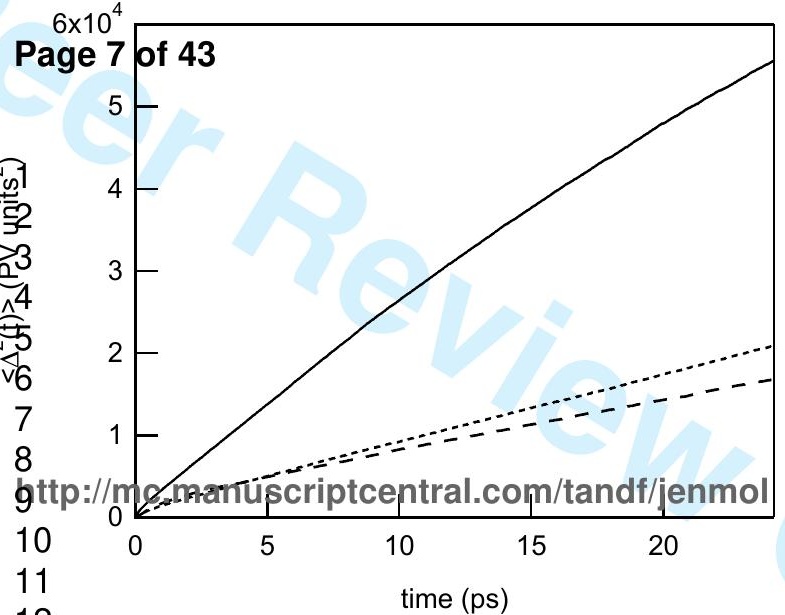




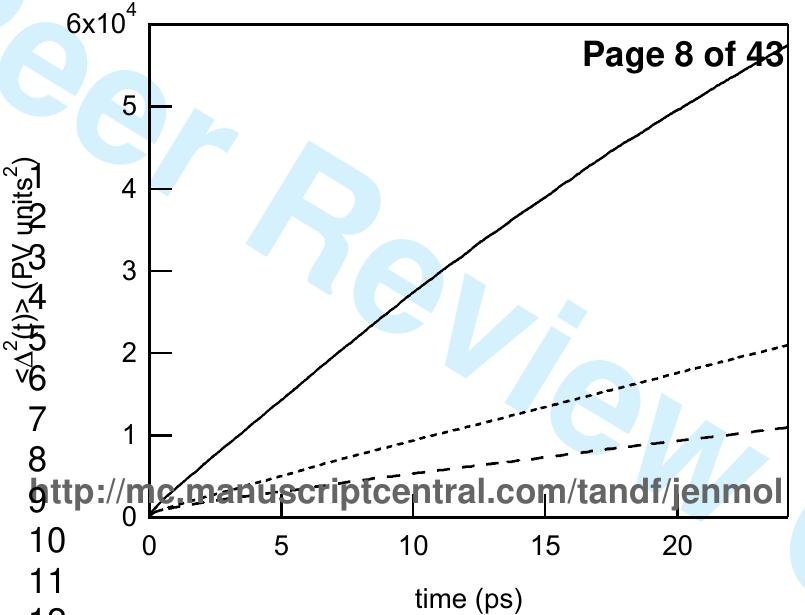


Charge Transport Along Phenylenevinylene Molecular Wires

Paulette Prins, Ferdinand C. Grozema, Laurens D. A. Siebbeles

Opto-electronic Materials, DelftChemTech

Delft University of Technology

Julianalaan 136, 2628 BL, Delft

The Netherlands 


\section{Introduction}

According to the predictions of Gordon Moore in 1965 the number of transistors per square centimetre in an integrated circuit doubles every 18 months.[1] This requires that the size of the transistors and the interconnecting wires decrease at the same rate. Until now the miniaturization of electronic components has been achieved by photolithographic techniques leading to the present-day integrated circuits where the width of the interconnecting copper wires is approximately $90 \mathrm{~nm}$. If the miniaturization continues at the rate predicted by Moore's law, the dimensions of the wires decrease to approximately $10 \mathrm{~nm}$ in the near future, approaching the fundamental size limits of photolithography.

Because of the limitations of conventional techniques it is of considerable interest to study the smallest components that are likely to be functional; i.e. components consisting of single molecules or assemblies of a few molecules.[2,3] Conjugated organic molecules are attractive candidates for use as interconnecting wires in molecular scale electronics because the properties relevant to charge transport; e.g. the band-gap, the ionization potential and the excited state properties, can be tuned by modifications in the molecular structure.[4-7] Additionally, synthetic organic chemistry techniques can be used to introduce functional units that can aid the assembly of conjugated molecules into supra-molecular structures by molecular recognition. This opens an interesting perspective in which electronic circuits are constructed by self-assembly rather than by external manipulation of the components.[8,9]

Recently, it has become possible to measure the conductance of single organic molecules between metal electrodes; e.g. in a break junction[10] or between mercury drops.[11] These methods provide insight in the transport of charges through the molecule, however, the results of such measurements are to a large extent determined by effects related to the contacts between electrodes and the molecule. In many cases the conductance can be considered to occur via a bridge-mediated superexchange mechanism in which the charge 
tunnels from one electrode to the other, without becoming localized on the molecular bridge.[12] [13] Hence, these measurements do not provide information about the mobility of charges that are localized on the molecule. However, the mobility of charges on isolated molecules can be measured by pulse-radiolysis time-resolved microwave conductivity (PRTRMC) experiments.[14,15] In this case, irradiation with high-energy electrons is used to generate charges on oligomer or polymer chains in solution, rather than by injecting them using electrodes. The mobility of charges moving along these molecular wires is determined without the use of electrodes by measuring the absorption of microwaves. In this way, information is obtained on the intrinsic charge transport properties of the molecular wires and effects caused by the presence of electrodes do not play any role. PR-TRMC measurements have been performed for a variety of conjugated polymers and it has been found that the mobility of charges along isolated conjugated polymer chains depends strongly on the structure of the backbone.[16-19]

In order to interpret and exploit the experimental data available from the measurements mentioned above it is essential to have a detailed theoretical framework describing charge transport along molecular wires.[20] The elementary processes in charge transport along conjugated polymers are similar to two-centre charge transfer reactions[21] where the efficiency of transfer depends on the energy difference and the electronic coupling (charge transfer integral) between the sites involved.[22-24] Charge transport along a molecular wire is more complicated, since the efficiency of the transport is influenced by all site energies and charge transfer integrals along the chain. The motion of the charge depends strongly on the degree of structural disorder along the chain, such as torsion between molecular units in a polymer.[17] Structural fluctuations in a molecular wire can lead to disorder in the energies of the charge at different sites as well as in the charge transfer 
integrals. To describe charge transport along molecular wires a model is required that contains detailed information on the structural fluctuations and the effects on charge transport.

Several theoretical models have been put forward for the description of experiments in which charge transport though a single molecule between metal electrodes is measured.[12,13,25] In this paper a model is presented to describe the mobility of charges moving along isolated molecular wires. The results can be compared with mobility measurements in which the charge is localised on the molecular wire, such as the PR-TRMC measurements mentioned above. The model is based on the tight-binding approximation and includes a classical description of the effects of structural fluctuations. The present model also includes dynamic disorder in contrast to previous work[17], where only static disorder has been considered. The theoretical framework is used to study the mobility of excess positive or negative charges along poly-phenylenevinylene (PPV) chains with variations in the number of alkoxy side chains. The calculations presented are used to illustrate how differences in the molecular structure and dynamics influence the efficiency of charge transport. 


\section{Theoretical Methodology}

\subsection{Model for charge carrier mobility based on tight-binding method}

The mean drift velocity of charge carriers moving on a molecular wire along the direction of an external electric field, $E(t)=E_{0} \cos (\omega t)$, is in the limit of linear response given by

$$
v(t)=\mu_{r}(\omega) E_{0} \cos (\omega t)+\mu_{i}(\omega) E_{0} \sin (\omega t)
$$

with $E_{0}$ the amplitude and $\omega$ the (radial) frequency of the electric field. The components of the charge carrier velocity in-phase and out-of-phase with the electric field are proportional to the real $\left(\mu_{r}(\omega)\right)$ and the imaginary part $\left(\mu_{i}(\omega)\right)$ of the complex mobility, respectively. According to the formulation by Kubo, the one-dimensional complex mobility is given by[2629]

$$
\mu(\omega)=-\frac{e \omega^{2}}{2 k_{B} T} \int_{0}^{\infty}\left\langle(x(t)-x)^{2}\right\rangle \mathrm{e}^{-i \omega t} d t
$$

with $e$ the elementary charge, $k_{B}$ Boltzmann's constant, $T$ the temperature and $\left\langle(x(t)-x)^{2}\right\rangle$ the mean square displacement of charge carriers in thermal equilibrium according to a Boltzmann distribution. An implicit convergence factor $e^{-\varepsilon t}(\lim \varepsilon \rightarrow 0)$ is understood in the integral.[27,28,30] The mean square displacement in equation (2) is evaluated by using the Heisenberg representation for the time-dependent position operator; i.e. $x(t)=\mathrm{e}^{i H t / \mathrm{h}} x \mathrm{e}^{-i H t / \mathrm{h}}$.[31] The Hamiltonian, $H$, depends not only on the coordinates of the 


\section{Page 15 of 43}

charge carrier, but also on those of the nuclei, or equivalently, on the static and dynamic structural fluctuations in the molecular wire.

$\underline{\text { In the remainder of this Section a general model to describe the mobility of charges on a }}$ molecular wire is presented and discussed. As outlined in Sections 2.2. and 2.3, a simplified form of the model is used to describe charge transport along phenylenevinylene molecular wires. The simplification described in sections 2.2. and 2.3. involves the neglect of all nuclear degrees of freedom other than the torsional motions of the phenyl groups, which are described classically.

In general a charge carrier on a molecular wire can be, described as a superposition of Deleted: The Deleted: is electronic states, $\left|\varphi_{s}^{\kappa}\right\rangle$, that are localized on the molecular units, $s$, in a wire. The index $\kappa$ denotes a particular realisation of the structural fluctuations in the molecular wire. Effects of nuclear vibrations are taken into account by vibrational states, $\left|\chi_{s}^{\kappa}\right\rangle$, associated with a charge at site $s$. The wave function of a charged molecular wire can then be written as

$$
\left|\Phi_{\kappa}\right\rangle=\sum_{s} a_{s}^{\kappa}\left|\varphi_{s}^{\kappa}\right\rangle\left|\chi_{s}^{\kappa}\right\rangle \equiv \sum_{s} a_{s}^{\kappa}|s, \kappa\rangle
$$

with $a_{s}^{\kappa}$ the expansion coefficient of the state $|s, \kappa\rangle$, which describes a charge localized at site $s$ in a molecular wire with a nuclear conformation and a vibrational state labelled with the index $\kappa$. The equilibrium density operator corresponds to a Boltzmann distribution; i.e.

$$
\rho=\sum_{\kappa} P_{\kappa}\left|\Phi_{\kappa}\right\rangle\left\langle\Phi_{\kappa}\right|
$$

with 


$$
P_{\kappa}=\frac{\mathrm{e}^{-E_{\kappa} / k_{B} T}}{\sum_{\kappa} \mathrm{e}^{-E_{\kappa} / k_{B} T}}
$$

and $E_{\kappa}$ the total energy of the state $\left|\Phi_{\kappa}\right\rangle$. The mean square displacement is obtained by averaging the square displacement over the density distribution of the charge. Combination of equations (3) and (4) then gives

$$
\begin{aligned}
& \left\langle(x(t)-x)^{2}\right\rangle=\operatorname{Tr}\left[(x(t)-x)^{2} \rho\right]=\sum_{\kappa} P_{\kappa}\left\langle\Phi_{\kappa}\left|(x(t)-x)^{2}\right| \Phi_{\kappa}\right\rangle \\
& =\sum_{\kappa} \sum_{s} \sum_{s^{\prime}} P_{\kappa} a_{s}^{\kappa}\left(a_{s^{\prime}}^{\kappa}\right)^{*}\left\langle s^{\prime}, \kappa\left|\mathrm{e}^{i H t / \mathrm{h}} x^{2} \mathrm{e}^{-i H t / \mathrm{h}}\right| s, \kappa\right\rangle \\
& \quad-\sum_{\kappa} \sum_{s} \sum_{s^{\prime}} P_{\kappa} a_{s}^{\kappa}\left(a_{s^{\prime}}^{\kappa}\right)^{*}\left[\left\langle s^{\prime}, \kappa\left|x \mathrm{e}^{i H t / \mathrm{h}} x \mathrm{e}^{-i H t / \mathrm{h}}\right| s, \kappa\right\rangle+\left\langle s^{\prime}, \kappa\left|\mathrm{e}^{i H t / \mathrm{h}} x \mathrm{e}^{-i H t / \mathrm{h}} x\right| s, \kappa\right\rangle\right] \\
& \quad+\sum_{\kappa} \sum_{s} P_{s^{\prime}} P_{\kappa} a_{s}^{\kappa}\left(a_{s^{\prime}}^{\kappa}\right)^{*}\left\langle s^{\prime}, \kappa\left|x^{2}\right| s, \kappa\right\rangle
\end{aligned}
$$

Since the states $|s, \kappa\rangle$ describe a charge localized at site $s$ and the vibrational states are taken orthogonal, the matrix elements of the position operator can to a good approximation be taken equal to $\left\langle s, \kappa|x| s^{\prime}, \kappa^{\prime}\right\rangle=s \lambda \delta_{s s^{\prime}} \delta_{\kappa \kappa^{\prime}}$ with $\lambda$ the distance between the sites. Using this relation, together with the identity operator expressed in terms of the localized charge carrier states; i.e. $U=\sum_{\kappa} \sum_{s}|s, \kappa\rangle\langle s, \kappa|$, equation (5) can be rewritten as 


$$
\begin{aligned}
& \left\langle(x(t)-x)^{2}\right\rangle= \\
& \sum_{\kappa, \kappa^{\prime \prime}} \sum_{s, s^{\prime}, s^{\prime \prime}} P_{\kappa} a_{s}^{\kappa}\left(a_{s^{\prime}}^{\kappa}\right)^{*} \lambda^{2}\left[\left(s^{\prime \prime}\right)^{2}-s^{\prime \prime}\left(s+s^{\prime}\right)\right]\left\langle s^{\prime}, \kappa\left|\mathrm{e}^{i H t / \mathrm{h}}\right| s^{\prime \prime}, \kappa^{\prime \prime}\right\rangle\left\langle s^{\prime \prime}, \kappa^{\prime \prime}\left|\mathrm{e}^{-i H t / \mathrm{h}}\right| s, \kappa\right\rangle \\
& \quad+\sum_{\kappa} \sum_{s} P_{\kappa} a_{s}^{\kappa}\left(a_{s}^{\kappa}\right)^{*} \lambda^{2} s^{2}
\end{aligned}
$$

The factor $\left\langle s^{\prime \prime}, \kappa^{\prime \prime}\left|\mathrm{e}^{-i H t / h}\right| s, \kappa\right\rangle$ in equation (6) is the coefficient of the state $\left|s^{\prime \prime}, \kappa^{\prime \prime}\right\rangle$ at time $t$, of a charge that was initially $(t=0)$ in the state $|s, \kappa\rangle$, localized at $s$. Analogously, the factor $\left\langle s^{\prime}, \kappa\left|\mathrm{e}^{i H t / h}\right| s^{\prime \prime}, \kappa^{\prime \prime}\right\rangle$ is the complex conjugate of the coefficient of $\left|s^{\prime \prime}, \kappa^{\prime \prime}\right\rangle$ for a charge initially localized at $s^{\prime}$ in the state $\left|s^{\prime}, \kappa\right\rangle$. Therefore, these coefficients correspond to charge migration from different initial sites to a common final site. The summation over $\kappa$ in equation (6) leads to averaging of the products of the coefficients for these different paths over realizations of molecular wires with distinct static and dynamic structural fluctuations. The different structural conformations and fluctuations will cause the phase of these expansion coefficients to vary from one wire to another. As a consequence the averaging over $\kappa$ will cause the terms for which $s \neq s^{\prime}$ in equation (6) to vanish on a timescale referred to as the dephasing (or decoherence) time. This dephasing time is determined by the amplitude and frequency of the fluctuations. The vanishing of the average of the cross-product terms with $s \neq s^{\prime}$ in equation (6) is analogous to the relaxation of the off-diagonal elements of the density matrix (in terms of localized states) of a particle moving under the influence of stochastic fluctuations due to interactions with a heat bath.[32-35] In summary, due to the effects of structural fluctuations 
the terms in equation (6) for which $s \neq s^{\prime}$ can often to a good approximation be neglected, so that equation (6) reduces to

$\left\langle(x(t)-x)^{2}\right\rangle=\sum_{\kappa, \kappa^{\prime \prime}} \sum_{s, s^{\prime \prime}} P_{\kappa}\left|a_{s}^{\kappa}\right|^{2} \lambda^{2}\left(s^{\prime \prime}-s\right)^{2}\left|\left\langle s^{\prime \prime}, \kappa^{\prime \prime}\left|\mathrm{e}^{-i H t / \mathrm{h}}\right| s, \kappa\right\rangle\right|^{2}$

Equation (7) describes the mean square displacement of charges that are initially localized at sites $s$, which migrate during a time interval $t$ to other sites $s^{\prime \prime}$ with a probability equal to

$$
\left|\left\langle s^{\prime \prime}, \kappa^{\prime \prime} \mathrm{e}^{-i H t / \mathrm{h}} \mid s, \kappa\right\rangle\right|^{2}
$$

At low temperatures structural fluctuations are less pronounced and the averaging over different molecular wires in equation (6) may not lead to cancellation of terms for which $s \neq s^{\prime}$. In this case the simplified equation (7) is not valid. At high frequencies of the external electric field, the integral in equation (2) is mainly determined by short times $(\sim 2 \pi / \omega)$ at which the average contribution of terms $s \neq s^{\prime}$ in equation (6) may not be negligible. In this case the simplification to equation (7) cannot be made.

In what follows it is assumed that fluctuations in the system lead to sufficient dephasing, so that the mean square displacement can be calculated according to equation (7). In the case of normal Gaussian diffusion the mean square displacement eventually increases linearly with time, according to

$$
\left\langle(x(t)-x)^{2}\right\rangle=2 D t
$$

with $D$ the diffusion constant. Substitution of equation (8a) into equation (2) gives the Einstein relation for the mobility 


$$
\mu=\frac{e}{k_{B} T} D
$$

The mobility in equation (8b) is frequency independent and the imaginary component is zero. It should be noted, that structural defects in a material can lead to barriers for charge transport. In that case the mean square displacement increases sublinearly with time and cannot be described by equation (8a). As a consequence barriers cause the mobility to be frequency dependent and to consist of non-zero real and imaginary components.

\subsection{Hybrid quantum/classical description of charge and nuclear dynamics}

The mobility of charge carriers is obtained from equation (8), by numerical simulation of the mean square displacement as defined in equation (7), where the charge carrier is described quantum mechanically by the tight-binding method including only interactions between neighbouring units. The relevant nuclear motions are often sufficiently slow so that they can, to a good approximation, be treated classically. In this case the Hamiltonian of the charge is given by

$$
H_{q}^{K}(t)=\sum_{s} \varepsilon_{s}^{K}(t) a_{s}^{+} a_{s}+\sum_{s} \sum_{s^{\prime}=s \pm 1} J_{s^{\prime}, s}^{K}(t) a_{s^{\prime}}^{+} a_{s}
$$

In equation (9) $a_{s}^{+}$and $a_{s}$ are the creation and annihilation operators of a charge at site $s$, $\varepsilon_{s}^{\kappa}(t)=\left\langle\varphi_{s}^{\kappa}\left|H_{q}^{\kappa}(t)\right| \varphi_{s}^{\kappa}\right\rangle$ is the site-energy of a charge and $J_{s^{\prime}, s}^{\kappa}(t)=\left\langle\varphi_{s^{\prime}}^{\kappa}\left|H_{q}^{\kappa}(t)\right| \varphi_{s}^{\kappa}\right\rangle$ is the charge transfer (bandwidth) integral. The site-energies can vary with time, since the energy of a charge at a site depends on the fluctuating molecular structure. The charge transfer integrals

Deleted: The time-dependence of these parameters is due to their dependence on the nuclear

coordinates, which in turn depend on time. depend on the mutual orientation of adjacent molecular units, which can also fluctuate during 
time. The time-propagation of the nuclear coordinates is described by the classical equations of motion with a potential that is in part determined by the distribution of the charge in the system; i.e. by the wave function $\left|\Phi_{\kappa}\right\rangle$ in equation (3). The mean square displacement in equation (7) can now be obtained by propagation of wave functions, which are initially localised at a single site $s$; i.e.

$$
\left|\psi_{\kappa}(t ; s)\right\rangle=\sum_{s^{\prime \prime}} c_{s^{\prime \prime}}^{\kappa}(t ; s)\left|\varphi_{s^{\prime \prime}}^{\kappa}\right\rangle
$$

with the initial condition $c_{s^{\prime \prime}}^{\kappa}(t=0 ; s)=1$ for $s^{\prime \prime}=s$ and zero for all other sites. Note, that the time-dependent wave functions defined in equation (10) are used to calculate the mean square displacement. The wave functions $\left|\psi_{\kappa}\right\rangle$ in equation (10) are distinct from the wave functions $\left|\Phi_{\kappa}\right\rangle$ in equation (3), which are stationary states describing the charged molecular wires. The time-dependent coefficients $c_{s^{\prime \prime}}^{\kappa}(t ; s)=\left\langle\varphi_{s^{\prime \prime}}^{\kappa}\left|e^{-i H_{q}^{\kappa}(t) t / \mathrm{h}}\right| \varphi_{s}^{\kappa}\right\rangle$ are obtained numerically by integration of the first-order differential equations that follow from the time-dependent Schrödinger equation

$$
i \mathrm{~h} \mathbf{S}^{\kappa} \frac{\partial \mathbf{c}^{\kappa}}{\partial t}=\mathbf{H}_{q}^{\kappa}(t) \mathbf{c}^{\kappa}
$$

with $\mathbf{c}^{\kappa}$ the vector containing the coefficients $c_{s "}^{\kappa}(t ; s)$. Note, that the spatial overlap matrix $\mathbf{S}^{\kappa}\left(S_{s^{\prime} s}^{\kappa}=\left\langle\varphi_{s^{\prime}}^{\kappa} \mid \varphi_{s}^{\kappa}\right\rangle\right)$ is explicitly taken into account. The effect of the spatial overlap can be incorporated into an effective Hamiltonian matrix $\mathbf{H}_{q, e f f}^{\kappa}(t)$ so that to a good approximation[36,37] 


$$
i \mathrm{~h} \frac{\partial \mathbf{c}^{\kappa}}{\partial t}=\mathbf{H}_{q, e f f}^{\kappa}(t) \mathbf{c}^{\kappa}
$$

The diagonal matrix elements of $\mathbf{H}_{q, \text { eff }}^{\kappa}$ are identical to the site energies $\varepsilon_{s}^{\kappa}$ in equation (9). When only nearest neighbour interactions are taken into account the non-zero off-diagonal matrix elements of $\mathbf{H}_{q, \text { eff }}^{\kappa}$ are equal to the effective charge transfer integrals given by[36,37]

$$
\left(\mathbf{H}_{q, e f f}^{\kappa}\right)_{s^{\prime}, s} \equiv\left(J_{e f f}^{\kappa}\right)_{s^{\prime}, s}=\left\langle\varphi_{s^{\prime}}^{\kappa}\left|H_{q}^{\kappa}\right| \varphi_{s}^{\kappa}\right\rangle-\frac{1}{2} S_{s^{\prime}, s}^{\kappa}\left(\varepsilon_{s^{\prime}}^{\kappa}+\varepsilon_{s}^{\kappa}\right) .
$$

Deleted account by using the time-dependent self-consistent field (TDSCF) method.[38] Following the TDSCF method, equation (11b), and the classical equations of motion for the nuclei, are propagated during small time steps. Each time step is taken sufficiently small so that the wave function of the charge can to a good approximation be propagated by assuming the nuclear coordinates to be fixed during a time step. The nuclei are propagated during the same time step, After a time step, the effect of the variation of the nuclear coordinates on the charge, is taken into account by adjusting the Hamiltonian of the charge according to the new nuclear coordinates. This procedure is repeated until a preset final time has been reached. As follows from equation (7), the mean square displacement can now be obtained from the timeDeleted: is Deleted: sufficiently Deleted: for Deleted: which Deleted: the nuclei can be considered fixed

Deleted: and the effective Hamiltonian can be taken constant

$$
\left\langle(x(t)-x)^{2}\right\rangle=\left\langle\sum_{s^{\prime \prime}, s} f(s) \lambda^{2}\left(s^{\prime \prime}-s\right)^{2}\left|c_{s^{\prime \prime}}(t ; s)\right|^{2}\right\rangle .
$$


The brackets at the right-hand side indicate averaging over different realizations of the structural fluctuations and $f(s)$ denotes the charge density at site $s$.

\subsection{Calculation of parameters for phenylenevinylene wires}

Phenylenevinylene molecular wires consist of an alternating sequence of phenylene and vinylene units. For a description of hole transport, the site-energies in equations (9) and (11c) are taken equal to the ionization energies of these molecular units, while for electron transport the electron affinities are used. As discussed below the site-energies for a hole or an excess electron are significantly lower at the phenyl unit than at the vinyl moiety. Hence, the hole and excess electron states are mainly composed of states on the phenyl rings. Therefore, the charge is initially taken to be localized on a phenyl ring. Effects of structural fluctuations on the site-energies are not taken into account. The effective charge transfer integral in equation (11c) for adjacent phenyl and vinyl units depends predominantly on the dihedral (torsion) angle between these moieties. Since other nuclear degrees of freedom have a much less pronounced effect on the charge transfer integrals, only the effects of torsional motion of the phenyl units in a molecular wire are taken into account. The dihedral angles between adjacent phenyl and vinyl units are distributed according to a Boltzmann distribution:

$$
P\left(\Theta_{s}-\Theta_{s-1}\right)=\frac{e^{-\frac{E_{t o r}\left(\Theta_{s}-\Theta_{s-1}\right)}{k_{B} T}}}{\int_{0}^{2 \pi} e^{-\frac{E_{t o r}\left(\Theta^{\prime}\right)}{k_{B} T}} d \Theta^{\prime}},
$$

where $E_{t o r}\left(\Theta_{s}-\Theta_{s-1}\right)$ is the potential energy for torsion around the bond between a phenyl and a vinyl unit at sites $s$ and $s-1$, respectively The charge will be delocalized along the 


\section{Page 23 of 43}

molecular wire, so that the amount of charge on a single PV unit is negligible. Therefore the potential energy for a neutral PV molecule can be used for $E_{\text {tor }}$.

$\underline{\text { In a PPV chain the phenyl groups can rotate around the single bonds with adjacent vinyl }}$ units. To be able to compare the present calculated results with measured mobility values for charges on isolated PPV chains in dilute benzene solution (see Section 3.5), the effects of the solvent on the rotational motion of the phenyl units are taken into account. The effect of interactions with the solvent will cause the phenyl units to undergo a random diffusive rotational motion superimposed on the drift in the potential energy landscape for torsion. The change of the angles of the phenyl units in time are described by a combination of rotational drift and diffusion. During a small time step $v_{\checkmark} \Delta t_{2}$ the change of the angle $\Theta_{s}$ of a phenyl unit with site index $s$ is given by[39]

$$
\Delta \Theta_{s}=-\frac{D_{r o t}}{k_{B} T} \frac{\partial}{\partial \Theta_{s}}\left(E_{t o r}\left(\Theta_{s}-\Theta_{s-1}\right)+E_{t o r}\left(\Theta_{s+1}-\Theta_{s}\right)\right) \Delta t+\Delta \Theta_{s, \text { diff }} .
$$

The first term is the rotational drift due to the torsion potential resulting from interaction with both neighbouring vinyl units, while the second term accounts for the random diffusive rotation. The latter term is calculated according to $\Delta \Theta_{s, \text { diff }}=\sqrt{24 D_{\text {rot }} \Delta t}$ Ran, with Ran a uniformly distributed random number in the interval $[-1 / 2,1 / 2]$, so that $\left\langle\Delta \Theta_{s, \text { diff }}^{2}\right\rangle=2 D_{\text {rot }} \Delta t$ with $D_{r o t}=1 / 2 \tau_{r o t}$ and $\tau_{r o t}$ the diffusional rotation time of a phenyl unit. The diffusional rotation time is defined as the time interval after which the root mean square variation of the angle of a phenyl unit equals one radian.

The effective charge transfer integrals (see equation 11c) are calculated for different dihedral angles, using density functional theory (DFT) in the program ADF[40] with fragment orbitals on the phenyl and vinyl units, according to the procedure described in ref.[37]. In this

Deleted: orientation
Deleted: is
Deleted: $(\Delta$
Deleted: $t)$
Deleted: $s$
Deleted: is

Deleted: 
way, the charge transfer integral is directly obtained as the off-diagonal matrix element of the Kohn-Sham Hamiltonian matrix.[37] This method of calculating the charge transfer integral gives direct and exact results, and does not rely on the assumption of zero spatial overlap between the molecules as made when the charge transfer integral is obtained from the orbital splitting. The charge transfer integrals for hole transport correspond to the off-diagonal matrix elements of the Kohn-Sham Hamiltonian involving the highest occupied molecular orbitals (HOMOs) on the phenyl and vinyl units. For excess electron transport the charge transfer integral was obtained from the lowest-unoccupied molecular orbitals (LUMOs). The calculations were performed using the Becke-Perdew gradient-corrected exchange correlation functional and a double- $\zeta$ plus polarization (DZP) basis set consisting of Slater-type functions.[41]

The torsion potential and the geometry of the phenylenevinylene unit are obtained by optimizing the geometry at the Hartree-Fock level while restraining the dihedral angle between the phenyl and the vinyl, using a cc-pVDZ basis set in the program Gaussian.[42]. Subsequently, the relative potential energies for each angle were calculated by second order Møller-Plesset perturbation theory (MP2).

The wave function of the charge and the conformation of the chain are propagated with a time step of one atomic unit $\left(2.4189 \times 10^{-17}\right.$ seconds) using equations (11b) and (14), respectively. The charge is initially localized on a phenyl group in the middle of a chain with sufficient length, such that the chain ends do not affect the mean square displacement on the time scale of the simulation. The mean square displacement of the charge carriers is obtained by averaging over a few hundred realizations of the initial torsion angles and their variation during time. 


\section{Results and Discussion}

\subsection{Parameters for charge transport calculations}

Charge transport calculations have been performed for three PPV derivatives with zero, one and two alkoxy side-chains on the phenyl group. The chemical structures of the model compounds of these three derivatives are shown in Figure 1. In order to gain insight in the

effect of the presence of side-chains on the conformation of the polymer chain the potential

Deleted: torsion energy for torsion around the bond connecting the phenyl and vinyl unit in a neutral styrene molecule was calculated using MP2, as discussed in section 2.3. The results are shown in Figure 2. For unsubstituted PPV the potential energy curve is symmetric around 90 degrees. The potential energy is minimum for a planar conformation and increases with $0.13 \mathrm{eV}$ when going to a perpendicular geometry. The potential energy is very close to the minimum value for angles up to approximately 30 degrees. The potential energy for PPV-ma (the PPV derivative with one alkoxy substituent) is not symmetric around 90 degrees, as a result of the loss of symmetry by the presence of the alkoxy substituent. The potential energy profile for PPV-ma is maximum at an angle of 105 degrees, while the energy is minimum at an angle of 30 degrees. The deviation from a planar conformation is attributed to steric hindrance between the alkoxy substituent and the hydrogen atom on the vinyl-group. At an angle of 30 degrees a balance is found between minimizing the steric hindrance and maximizing the conjugation. A local minimum is found at 180 degrees at an energy $0.05 \mathrm{eV}$ higher than at the absolute minimum, which is due to the increased steric hindrance of the alkoxy side-chain and the vinyl unit. For PPV-da (the PPV derivative with two alkoxy substituents) the substitution pattern is more symmetric and the potential energy is minimum for the planar conformation. The potential energy increases by $0.18 \mathrm{eV}$ to reach a maximum at a perpendicular geometry. 
The effective charge transfer integrals to be used in charge transport calculations were calculated using the fragment orbital approach as discussed in Section 2. Figure 3 shows the angular dependence of the effective charge transfer integrals for hole transport, as calculated from the HOMO orbitals on the phenyl and vinyl units, for the three PPV derivatives considered here. In all cases the maximum is found at a planar geometry where the interaction between the $\pi$-systems of the two units is optimal. The shape of the coupling is close to a cosine for all three PPV derivatives. The charge transfer integrals for PPV-ma and PPV-da are almost a factor two smaller than for non-substituted PPV. This can be understood by examining the spatial extent of the HOMO orbitals on the phenyl part of the model system. The density of the HOMOs on the carbons adjacent to the alkoxy side-chains is increased, which leads to a reduced density on the carbon atom that is connected to the vinyl moiety. This gives rise to the lower effective charge transfer integral obtained for the alkoxy substituted PPVs.

The effective charge transfer integrals between the lowest unoccupied molecular orbitals (LUMO) of the phenyl and vinyl units are used to describe electron transport. The results for the three PPV derivatives are shown in Figure 4. As for the HOMOs, the effective charge transfer integrals for the LUMOs are maximal for a planar conformation, but deviate from a cosine shape. The influence of alkoxy substituents on the charge transfer integrals between the LUMOs is smaller than found for the HOMO orbitals. This is consistent with previous calculations where it was shown that the presence of alkoxy substituents has a considerable localizing effect on positive charges on PV chains,[43] whereas the effect on the distribution of a negative charge is much smaller.[44] Figures 3 and 4 show that the charge transfer integrals obtained from the HOMOs and LUMOs have comparable values. On basis of this result it is to be expected that phenylenevinylene molecular wires can act both as electron and as hole conductor. 


\subsection{Effect of parameters on charge carrier mobility}

The magnitude of the mobility of charges along PPV chains calculated using the model presented in Section 2 is dependent on four parameters. Apart from the potential energy surface and the effective charge transfer integral that were discussed above, the mobility depends on the rotational diffusion time of the phenyl units $\left(\tau_{r o t}\right)$ and on the site energy difference between the vinyl and phenyl units. In order to gain insight in the effect of the individual parameters a series of calculations has been performed in which these parameters are varied systematically. In all calculations the temperature was taken equal to $293 \mathrm{~K}$.

Figure 5 shows the effect of the magnitude of the effective charge transfer integral on the mean square displacement. The dependence of the charge transfer integral on the torsion angle was taken identical to that calculated for unsubstituted PPV. The magnitude of the effective charge transfer integral was scaled such that it was equal to $0.225 \mathrm{eV}, 0.45 \mathrm{eV}$ or $0.90 \mathrm{eV}$ at zero angle. The potential energy for unsubstituted PPV was used together with the site energy difference for holes on the vinyl and phenyl units equal to $1.2 \mathrm{eV}$, as obtained from the ionization potentials of ethylene[45] and benzene[46]. The rotational diffusion time was taken to be 50 ps. The mean square displacement was obtained by propagation of the wave function of an initially localized charge, as discussed in Section 2. On a short time scale the mean square displacement is dominated by charges on the relatively planar parts of the chain, leading to the initial $(<1 \mathrm{ps})$ fast increase of the mean square displacement. At longer times the motion of the charges is determined by all dihedral angles resulting from the Boltzmann distribution in equation (13) and limited by large angles. After a few ps the motion of charges becomes diffusive and the mean square displacement increases linearly with time according to equation (8a). In this linear regime the diffusion coefficient can be obtained from the slope of the curves in Figure 5 and the charge carrier mobility can be calculated using 
equation (8b). The charge carrier mobilities obtained from the curves in Figure 5 are 29 $\mathrm{cm}^{2} / \mathrm{Vs}, 52 \mathrm{~cm}^{2} / \mathrm{Vs}$ and $90 \mathrm{~cm}^{2} / \mathrm{Vs}$ for magnitudes at zero angle of the effective charge transfer integral equal to $0.225 \mathrm{eV}, 0.45 \mathrm{eV}$ and $0.90 \mathrm{eV}$, respectively. These results show that the mobility increases almost linearly with the magnitude of the charge transfer integral. Note, that the effect of the magnitude of the charge transfer integral on the mobility is very distinct from the case of polaronic hopping transport between localized states[47,48] or the motion of charges under the influence of white-noise like dynamic fluctuations.[32,33,49] In the latter cases the mobility increases quadratically with the magnitude of the charge transfer integral. A linear increase of the mobility with the charge transfer integral is expected for band-like transport with a mean thermal velocity of the charge carriers proportional to the bandwidth and a mean free path for scattering which is independent of the velocity.[47] Further investigations are needed to explain the origin of the almost linear dependence of the mobility on the charge transfer integral, as obtained from the current calculations on PPV.

In order to verify the validity of neglecting the cross-product terms with $s \neq s^{\prime}$ in equation (6) the magnitude of these terms obtained by averaging over $10^{4}$ realizations of the diffusional rotational motion of the phenyl units was calculated for a few specific values of $s$ and $s^{\prime}$. The average value of the cross-product terms was found to rapidly oscillate with a period of the order of $10 \mathrm{fs}$ with an amplitude less than a few percent of the magnitude of the corresponding terms for which $s=s^{\prime}$. Hence, these cross-product terms are indeed negligible and the mobility can to a good approximation be calculated by taking equation (7) as a starting point.

In Figure 6 the mean square displacement is shown for different rotational diffusion times of the phenyl units. The calculations were performed with the potential energy curve and site energy difference for unsubstituted PPV, as specified above. The angular dependence of the effective charge transfer integral for holes on unsubstituted PPV was used with a 
scaling factor such that the magnitude at zero angle is equal to $0.45 \mathrm{eV}$. From the slopes of the curves in Figure 6 charge carrier mobilities equal to $104 \mathrm{~cm}^{2} / \mathrm{Vs}, 51 \mathrm{~cm}^{2} / \mathrm{Vs}$ and 28 $\mathrm{cm}^{2} / \mathrm{Vs}$ were obtained for rotational diffusion times of $10 \mathrm{ps}, 50 \mathrm{ps}$ and $100 \mathrm{ps}$, respectively. These results illustrate that charge transport is faster when the phenyl rings rotate more rapidly. This can be understood since charge transport is limited by the occurrence of dihedral angles near 90 degrees for which the charge transfer integrals are smallest. As the rotation time decreases such non-planar conformations change faster to a more planar conformation, which is favourable to charge transport.

The effect of the difference between the site energy for the vinyl and phenyl unit has also been studied. The rotational diffusion time was taken equal to $50 \mathrm{ps}$ and the other parameters were identical to those for the data in Figure 6. For site energy differences of 1.2 $\mathrm{eV}$ and $2.6 \mathrm{eV}$ the calculated charge carrier mobilities are $52 \mathrm{~cm}^{2} / \mathrm{Vs}$ and $38 \mathrm{~cm}^{2} / \mathrm{Vs}$, respectively. The mobility decreases as the site energy difference increases. This can be understood, since charge transfer between the phenyl units can be considered as a tunnelling process through the energetic barrier resulting from to the intermediate vinyl unit. This process becomes slower as the barrier for tunnelling increases.

\subsection{Hole mobility on PPV, PPV-ma and PPV-da}

The potential energy curves and charge transfer integrals for holes presented in Figures 2 and 3 were used to calculate the mean square displacement and the charge carrier mobility for holes along the three PPV derivatives shown in Figure 1. The potential energy profiles, the charge transfer integrals, the site energy at the phenyl units and the rotational diffusion time of the latter are different for these PPV derivatives. The site energies for holes were taken equal to the experimental ionization potentials of ethylene,[45] benzene,[46] methoxy-benzene[50] and di-methoxy benzene.[51] The differences between the site energies 
for the vinyl and phenyl units are then found to be $1.2 \mathrm{eV}, 2.3 \mathrm{eV}$ and $2.6 \mathrm{eV}$ for PPV, PPV$m a$ and PPV-da, respectively. The rotational diffusion times of the phenyl units were estimated from experimental rotational diffusion times of molecules of similar size. For unsubstituted PPV a rotational diffusion time of $10 \mathrm{ps}$ was taken which is of the same order of magnitude as the rotational diffusion time of a benzene molecule in liquid benzene.[52] Soluble alkoxy substituted PPVs of interest for applications in plastic electronic devices typically contain around 10 carbon atoms in the side chains. This leads to a considerably longer rotation time than for an unsubstituted benzene molecule. For PPV- $d a$ a rotational diffusion time of 200 ps was taken, which is a typical rotation time for molecules of similar size.[53] For PPV-ma an intermediate rotational diffusion time of 50 ps was assumed.

The simulated mean square displacement of holes is shown in Figure 7 for the three PPV derivatives mentioned above. The hole mobility is obtained from the slope of the curves at longer times and using equation (8). The mobility value of $179 \mathrm{~cm}^{2} / \mathrm{Vs}$ obtained for unsubstituted PPV is significantly higher than for the alkoxy substituted PPVs. This can be understood, since for unsubstituted PPV the charge transfer integral is larger than for the alkoxy substituted PPV derivatives. In addition the rotational diffusion time and the site energy difference are smallest for unsubstituted PPV. The hole mobilities for PPV-ma and PPV- $d a$ are found to be $78 \mathrm{~cm}^{2} / \mathrm{Vs}$ and $59 \mathrm{~cm}^{2} / \mathrm{Vs}$, respectively. Although the charge transfer integrals for PPV-ma and PPV-da are similar (see Figure 3) the hole mobility found for PPV$d a$ is smaller. This is due to the larger rotational diffusion time of the di-alkoxy substituted phenyl units and the somewhat larger site energy difference for PPV-da.

\subsection{Electron mobility on PPV, PPV-ma and PPV-da}

The mean square displacement and mobility for excess electrons along the three PPV derivatives were calculated using the potential energy curves in Figure 2 and charge transfer 
integrals for electrons in Figure 4. The rotational diffusion times of the phenyl units of the different PPV derivatives were estimated as discussed above. The differences between the site energy of an excess electron at the vinyl and the phenyl units was taken equal to the difference between the electron affinities calculated with DFT. The difference of the site energies for an excess electron at the vinyl and the phenyl units found in this way are $0.9 \mathrm{eV}$ for unsubstituted PPV, $1.0 \mathrm{eV}$ for PPV-ma and $1.1 \mathrm{eV}$ for PPV-da.

The mean square displacement for electrons on the three PPV derivatives is shown in Figure 8. The electron mobilities obtained from equation (8) with the slope of the curves at longer times in Figure 8, are $36 \mathrm{~cm}^{2} / \mathrm{Vs}, 76 \mathrm{~cm}^{2} / \mathrm{Vs}$ and $181 \mathrm{~cm}^{2} / \mathrm{Vs}$ for PPV, PPV-ma and PPV-da, respectively. The magnitudes of the charge transfer integrals and the site energy differences are similar for electrons on the three PPV derivatives. Hence, the observed difference in electron mobility is mainly caused by the different rotational diffusion times used in the calculations.

\subsection{Comparison with experimental data}

The mobility of charges along isolated di-alkoxy substituted PPV chains in solution has been determined experimentally using the pulse-radiolysis time-resolved microwave conductivity (PR-TRMC) technique.[14,16,54] In these experiments, charge carriers are generated by irradiation of a dilute solution of PPV in the non-polar sovent benzene with 3 $\mathrm{MeV}$ electrons and the change in the microwave conductivity at a frequency of $34 \mathrm{GHz}$ is measured. The ac mobility values at $34 \mathrm{GHz}$ obtained from these experiments are in the order of a few tenths of $\mathrm{cm}^{2} / \mathrm{Vs}$. These experimental mobility values cannot be directly compared with the calculated values discussed in Sections 3.3 and 3.4. The PPVs used in the experiments inevitably contain a few percent of defects in the form of $e . g$. saturated carbon bonds, which disrupt the conjugated pi-system of the polymer chain. Such conjugation breaks 
act as barriers to charge transport and have a limiting effect on the mobility. The occurrence of chain ends or infinitely high barriers leads to zero dc mobility. However, at the experimental frequency of $34 \mathrm{GHz}$ the mobility is non-zero, since at higher frequency the charges can follow the oscillating field by moving between the conjugation breaks or chain ends.

The limiting effect of defects on the $34 \mathrm{GHz}$ mobility has been nicely illustrated by the experimental studies on di-alkoxy substituted PPV chains with varying fraction of conjugation breaks. The mobility is found to increase from $0.01 \mathrm{~cm}^{2} / \mathrm{Vs}$ to $0.46 \mathrm{~cm}^{2} / \mathrm{Vs}$ as the fraction of defects decreases from 30 percent to about 5 percent for the 'fully' conjugated polymer.[54] These experimental results could be reproduced by the theoretical methodology presented above, when the effects of the limited conjugation lengths were taken into account.[55] Hence, the theoretical model and the new results of the present work for infinite PPV chains with different substituents can be considered reliable.

\section{Summary and outlook}

A theoretical model to calculate the mobility of charges along polymer chains has been presented. The model is based on the tight-binding approximation and includes a classical description of the effects of the torsional motion of the units in the polymer chains. This torsional motion has a direct influence on the motion of the charge carrier through the dependence of the charge transfer integral between neighbouring units on the torsion angle between the units. The parameters necessary to calculate the charge carrier mobility are either obtained from electronic structure calculations or from experimental data.

The model has been used to simulate the motion of charges along isolated polyphenylenevinylene (PPV) chains. It was shown that the charge carrier mobility is affected by the torsional motion of the phenyl units. When these units rotate more slowly, larger inter-unit 
angles persist for a longer time, leading to lower charge carrier mobilities. Simulations have been performed for PPV derivatives with zero, one and two alkoxy side-chains. The presence of alkoxy substituents was found to affect the charge transfer integrals, the torsion potential energy surface and the energy differences along the chain. The effects mentioned above lead to considerable differences in the charge carrier mobility for the three PPV derivatives. The mobility for holes and electrons is highest for unsubstituted PPV and reduces by about a factor of two upon introduction of one substituent. The mobility for di-alkoxy substituted PPV is more than a factor of three lower than that found for unsubstituted PPV.

Experimental data on the ac charge carrier mobility along di-alkoxy substituted PPV chains can be reproduced by the theoretical model when effects of conjugation breaks along the PPV chains are taken into account. According to the analysis of the experimental data on basis of the theoretical model an intra-chain mobility value of $59 \mathrm{~cm}^{2} / \mathrm{Vs}$ would be attained in the absence of defects.

Although the mobilities obtained from the model presented in this paper give a reasonably adequate description of the experimental data, there are some effects that have been neglected. The most important approximations made in the present model involve the effects of static and dynamic disorder on the site energies and charge transfer integrals. In the present model only the effect of torsional motion of molecular units in the polymer on the charge transfer integrals was taken into account. However, many other structural degrees of freedom will affect both the site energies and the charge transfer integrals.

The rather limited description of the structural degrees of freedom has three related consequences. First of all, the motion of the charge will be influenced by structural degrees of freedom other than torsional motion. For example, structural fluctuations inside the sub-units making up the polymer chain can have a significant effect on the spatial distribution of the HOMO and LUMO orbitals in a unit. This may lead to changes in the amplitude of the wave 
function on the carbon atoms that connect adjacent units in the polymer chain, which influences the charge transfer integral and the consequently the charge carrier mobility. Secondly, the charge carrier may induce a geometrical distortion of the lattice around itself to reach a lower energy. This process of polaron formation can lead to charge transport by hopping between localised states and a reduction of the mobility. The third consequence of the limited description of the structural degrees of freedom is that the energy exchange between the charge carrier and the vibrations is very small. In reality the charge is coupled to a large bath of vibrations, so that the charge can easily exchange energy with vibrations. This is of particular relevance for simulations of the motion of charges in an external electric field. If a charge carrier is moving in an electric field it needs to dissipate the energy it gains from the applied field to the bath of vibrations. However, if the coupling with vibrations is too small or the bath of vibrations is very limited, the charge will not be able to dissipate its energy and will therefore not move in an external electric field. This leads to so-called Stark localization of the charge.

In order to arrive at a more complete description of charge transport along conjugated polymer chains more structural degrees of freedom should be included. Therefore it is of particular interest to identify the structural deformations that affect the motion of the charge most. The development of a general quantum mechanical model describing the energy exchange between a charge carrier and a bath of vibrations will be useful for simulations of charge transport in the presence of external electric fields. In particular in conductance measurements on nanoscale molecular electronic devices high electric field strengths are often used and in that case the limit of linear response considered in the present work may no longer be applicable.

The theoretical model presented in this work involves band-like transport along molecular wires with disorder in the charge transfer integrals. This model is adequate for the 
PPV derivatives considered in this work, since the charge transfer integrals are relatively large and strong localisation of the charge will not occur. It will however be of interest to extend the current model by inclusion of a more detailed description of charge-lattice interactions, so that the limit of hopping transport between localised states can also be described. Such a development will make the model applicable to a large range of molecular wires with different magnitudes of charge transfer integrals and varying degrees of structural disorder.

\section{References}

[1] G.E. Moore, Cramming more components onto integrated circuits. Electronics, $\mathbf{3 8}$, (1965).

[2] N. Hush, An overview of the first half-century of molecular electronics. Ann. NY Acad. Sci., 1006, 1-20 (2003).

[3] D.K. James, J.M. Tour, Molecular wires. Top. Curr. Chem., 257, $33-62$ (2005).

[4] S.-J. Chung, J.-I. Jin, K.-K. Kim, Novel PPV derivatives emitting light over a broad wavelength range. Adv. Mater., 9, 551-554 (1997).

[5] P.F. van Hutten, G. Hadziioannou. 2000. A model oligomer approach to semiconducting polymers. In: Hadziioannou G., van Hutten P.F., editors. Semiconducting polymers. Weinheim: Wiley-VCH.

[6] P.F. van Hutten, V.V. Krasnikov, G. Hadziioannou, A model oligomer approach to light-emitting semiconducting polymers. Acc. Chem. Res., 32, 257-265 (1999).

[7] F.C. Grozema, R. Telesca, J.G. Snijders, L.D.A. Siebbeles, Tuning of the excited state properties of phenylenevinylene oligomers: A time-dependent density functional theory study. J. Chem. Phys., 118, 9441-9446 (2003). 
[8] F.J.M. Hoeben, P. Jonkheijm, E.W. Meijer, A.P.H.J. Schenning, About supramolecular assemblies of pi-conjugated systems. Chem. Rev., 105, 1491-1546 (2005).

[9] J.A.A.W. Elemans, A.E. Rowan, R.J.M. Nolte, Mastering molecular matter. Supramolecular architectures by hierachical self-assembly. J. Mater. Chem., 13, 26612670 (2003).

[10] M.A. Reed, C. Zhou, C.J. Muller, T.P. Burgin, J.M. Tour, Conductance of a molecular junction. Science, 278, 252-254 (1997).

[11] K. Slowinski, K.Y. Fong, M. Majda, Mercury-mercury tunneling junctions. 1. Electron tunneling across symmetric and assymmetric alkanethiolate bilayers. J. Am. Chem. Soc., 121, 7257-7261 (1999).

[12] M.A. Ratner, J. Jortner. 1997. Molecular electronics: Some directions. In: Ratner M.A., Jortner J., editors. Molecular electronics. Oxford: Blackwell Science. pp 5-72.

[13] E.A. Weiss, M.R. Wasielewski, M.A. Ratner, Molecules as wires: Molecule assisted movement of charge and energy. Top. Curr. Chem., 257, 103-133 (2005).

[14] R.J.O.M. Hoofman, M.P. de Haas, L.D.A. Siebbeles, J.M. Warman, Highly mobile electrons and holes on isolated chains of the semiconducting polymer poly(phenylenevinylene). Nature, 392, 54-56 (1998).

[15] F.C. Grozema, R.J.O.M. Hoofman, L.P. Candeias, M.P. de Haas, J.M. Warman, L.D.A. Siebbeles, The Formation and Recombination Kinetics of Positively Charged Poly(phenylene vinylene) Chains in Pulse-Irradiated Dilute Solutions. J. Phys. Chem. A, 107, 5976-5986 (2003).

[16] F.C. Grozema, L.D.A. Siebbeles, J.M. Warman, S. Seki, S. Tagawa, U. Scherf, Hole conduction along molecular wires: sigma-bonded silicon versus pi-bond-conjugated carbon. Adv. Mater., 14, 228-231 (2002). 
[17] F.C. Grozema, P.T. van Duijnen, Y.A. Berlin, M.A. Ratner, L.D.A. Siebbeles, Intramolecular charge transport along isolated chains of conjugated polymers: Effect of torsional disorder and polymerization defects. J. Phys. Chem. B, 106, 7791-7795 (2002).

[18] P. Prins, L.P. Candeias, A.J.J.M. Van Breemen, J. Sweelssen, P.T. Herwig, H.F.M. Schoo, L.D.A. Siebbeles, Electron and hole dynamics on isolated chains of a solution processible poly(thienylene vinylene) derivative in dilute solution. Adv. Mater., 17, $718-723$ (2005).

[19] L.D.A. Siebbeles, F.C. Grozema, M.P. de Haas, J.M. Warman, Effect of backbone structure on charge transport along isolated conjugated polymer chains. Rad. Phys. Chem., 72, 85-91 (2005).

[20] D. Adams, M., L. Brus, C.E.D. Chidsey, S. Creager, C. Creutz, C.R. Kagan, P.V. Kamat, M. Lieberman, S. Lindsay, R.A. Marcus, R.M. Metzger, M.E. Michel-Beyerle, J.R. Miller, M.D. Newton, D.R. Rolison, O. Sankey, K.S. Schanze, J. Yardley, X. Zhu, Charge transfer on the nanoscale: current status. J. Phys. Chem. B, 107, 66686697 (2003).

[21] A. Nitzan, A relationship between electron-transfer rates and molecular conduction. $J$. Phys. Chem. A, 105, 2677-2679 (2001).

[22] G. Ashkenazi, R. Kosloff, M.A. Ratner, Photoexcited electron transfer: short-time dynamics and turnover control by dephasing, relaxation and mixing. J. Am. Chem. Soc., 121, 3386 (1999).

[23] W.B. Davis, M.A. Ratner, M.R. Wasielewski, Dependence of electron transfer dynamics in wire-like bridge molecules on donor-bridge energetics and electronic interactions. Chem. Phys., 281, 333-346 (2002). 
[24] G.L. Closs, J.R. Miller, Intramolecular long-distance electron transfer in organic molecules. Science, 240, 440 (1988).

[25] C. Chiorboli, M.T. Indelli, F. Scandola, Photoinduced electron/energy transfer across molecular bridges in binuclear metal complexes. Top. Curr. Chem., 257, 63-102 (2005).

[26] R. Kubo, Statistical-mechanical theory of irreversible processes I. General theory and simple applications to magnetic and conduction problems. J. Phys. Soc. Jap., 12, 570586 (1957).

[27] H. Scher, M. Lax, Stochastic transport in a disordered solid I. Theory. Phys. Rev. B, 7, 4491-4502 (1973).

[28] J.C. Dyre, T.B. Schrøder, Universality of AC conduction in disordered solids. Rev. Mod. Phys., 72, 873-892 (2000).

[29] U. Mizutani, Introduction to the Electron Theory of Metals. Cambridge University Press, Cambridge (2001)

[30] D.N. Zubarev, Nonequilibrum Statistical Thermodynamics. Plenum Press, New york (1974)

[31] G.C. Schatz, M.A. Ratner, Quantum Mechanics in Chemistry. Dover Publications, Mineola, N.Y., USA (2002)

[32] P. Reineker, Equations of motion for the moments of the coupled coherent and incoherent motion of triplet and singlet excitons. Z. Physik, 261, 187-190 (1973).

[33] E.A. Silinsh, Organic Molecular Crystals. Springer-Verlag, Berlin (1980)

[34] D.H. Dunlap, V.M. Kenkre, Exciton Dynamics in Molecular Crystals and Aggregates. Springer, Berlin (1982)

[35] V. May, O. Kühn, Charge and Energy Transfer Dynamics in Molecular Systems. Wiley, Berlin (2000) 
[36] M.D. Newton, Quantum chemical probes of electron-transfer kinetics: The nature of donor-acceptor interactions. Chem. Rev., 91, 767-792 (1991).

[37] K. Senthilkumar, F.C. Grozema, F.M. Bickelhaupt, L.D.A. Siebbeles, Charge transport in columnar stacked triphenylenes: Effects of conformational fluctuations on charge transfer integrals and site energies. J. Chem. Phys., 119, 9809-9817 (2003).

[38] R. Kosloff, Time-dependent Quantum-mechanical methods for molecular dynamics. $J$. Phys. Chem., 92, 2087 (1988).

[39] H. Risken, The Fokker-Planck Equation. Springer-Verlag, Berlin (1984)

[40] G. te Velde, F.M. Bickelhaupt, E.J. Baerends, C. Fonseca Guerra, S.J.A. van Gisbergen, J.G. Snijders, T. Ziegler, Chemistry with ADF. J. Comp. Chem., 22, 931967 (2001).

[41] J.G. Snijders, P. Vernooijs, E.J. Baerends, Roothaan-Hartree-Fock-Slater atomic wave functions. Atomic Data and Nuclear Data Tables, 26, 483-509 (1981).

[42] M.J. Frisch, G.W. Trucks, H.B. Schlegel, G.E. Scuseria, M.A. Robb, J.R. Cheeseman, V.G. Zakrzewski, J. Montgomery, J. A., R.E. Stratmann, J.C. Burant, S. Dapprich, J.M. Millam, A.D. Daniels, K.N. Kudin, M.C. Strain, O. Farkas, TomasiJ., V. Barone, M. Cossi, R. Cammi, B. Mennucci, C. Pomelli, C. Adamo, S. Clifford, J. Ochterski, G.A. Petersson, P.Y. Ayala, Q. Cui, K. Morokuma, D.K. Malick, A.D. Rabuck, K. Raghavachari, J.B. Foresman, J. Cioslowski, J.V. Ortiz, A.G. Baboul, B.B. Stefanov, G. Liu, A. Liashenko, P. Piskorz, I. Komaromi, R. Gomperts, R.L. Martin, D.J. Fox, T. Keith, M.A. Al-Laham, C.Y. Peng, A. Nanayakkara, C. Gonzalez, M. Challacombe, P.M.W. Gill, B. Johnson, W. Chen, M.W. Wong, J.L. Andres, C. Gonzalez, M. Head-Gordon, E.S. Replogle, J.A. Pople. 1998. Gaussian 98, Revision A.7. Pittsburgh PA: Gaussian Inc. 
[43] F.C. Grozema, L.P. Candeias, M. Swart, P.T. van Duijnen, J. Wildeman, G. Hadziioannou, L.D.A. Siebbeles, J.M. Warman, Theoretical and experimental studies of the opto-electronic properties of positively charged oligo(phenylene vinylene)s: Effects of chain length and alkoxy substitution. J. Chem. Phys., 117, 11366-11378 (2002).

[44] S. Fratiloiu, F.C. Grozema, L.D.A. Siebbeles, Optical properties and delocalization of excess negative charges on oligo(phenylenevinylene)s: A quantum chemical study. $J$. Phys. Chem. B, 109, 5644-5652 (2005).

[45] K. Ohno, K. Okamura, H. Yamakado, S. Hoshino, T. Takami, M. Yamauchi, Penning ionization of $\mathrm{HCHO}, \mathrm{CH} 2 \mathrm{CH} 2$, and $\mathrm{CH} 2 \mathrm{CHCHO}$ by collision with $\mathrm{He}^{*}(23 \mathrm{~S})$ metastable atoms. J. Phys. Chem., 99, 14247-14253 (1995).

[46] G.I. Nemeth, H.L. Selzle, E.W. Schlag, Magnetic ZEKE experiments with mass analysis. Chem. Phys. Lett., 215, 151-155 (1993).

[47] M. Pope, C.E. Swenberg, Electronic processes in organic crystals and polymers. Oxford University Press, Oxford (1999)

[48] T. Holstein, Studies of polaron motion. Part II, The small polaron. Ann. Phys., 8, 343389 (1959).

[49] M.A. Palenberg, R.J. Silbey, M. Malagoli, J.-L. Brédas, Almost temperature independent charge carrier mobilities in liquid crystals. J. Chem. Phys., 112, 1541 (2000).

[50] R.G. Cooks, M. Bertrand, J.H. Beynon, M.E. Rennekamp, D.W. Setser, Energy partitioning data as an ion structure probe. Substituted anisoles. J. Am. Chem. Soc., 95, 1732-1739 (1973). 
1

2

3

4

5

6

7

8

9

10

11

12

13

14

15

16

17

18

19

20

21

[51] G.M. Anderson III, P.A. Kollman, L. Domelsmith, N, K.N. Houk, Methoxy group nonplanarity in o-dimethoxybenzenes. Simple predictive models for conformation and rotational barriers in alkoxyaromatics. J. Am. Chem. Soc., 101, 2344-2352 (1979).

[52] R. Witt, L. Sturz, A. Dolle, F. Muller-Plathe, Molecular dynamics of benzene in neat liquid and a solution containing polystyrene. $13 \mathrm{C}$ nuclear magnetic relaxation and molecular dynamics simulation results. J. Phys. Chem. A, 104, 5716-5725 (2000).

[53] G.B. Dutt, G. Rama Krishna, S. Raman, Rotational dynamics of coumarins in nonassociative solvents: Point dipole versus extended charge distribution models of dielectric friction. J. Chem. Phys., 115, 4732-4741 (2001).

[54] L.P. Candeias, F.C. Grozema, G. Padmanaban, S. Ramakrishnan, L.D.A. Siebbeles, J.M. Warman, Positive charge carriers on isolated chains of MEH-PPV with broken conjugation: Optical absorption and mobility. J. Phys. Chem. B, 107, 1554-1558 (2002).

[55] P. Prins, F.C. Grozema, L.D.A. Siebbeles, submitted for publication. 


\section{Figure Captions}

Figure 1: Molecular structure of model structures for poly-phenylenevinylene (PPV), monoalkoxy substituted PPV (PPV-ma) and di-alkoxy substituted PPV (PPV-da).

Figure 2: Potential energy for neutral PV model systems as a function of the dihedral angle between the phenylene and vinylene units. Triangles: PPV, squares: PPV-ma, asterisks: PPVda.

Figure 3: Effective charge transfer integral for hole transport as a function of the dihedral angle between phenylene and vinylene units. Triangles: PPV, squares: PPV-ma, asterisks: PPV-da.

Figure 4: Effective charge transfer integral for electron transport as a function of the dihedral angle between phenylene and vinylene units. Triangles: PPV, squares: PPV-ma, asterisks: PPV-da

Figure 5: Mean square displacement as a function of time for different magnitudes of the effective charge transfer integral: $0.225 \mathrm{eV}, 0.45 \mathrm{eV}$ and $0.90 \mathrm{eV}$ (at zero angle) from bottom to top.

Figure 6: Mean square displacement as a function of time for different rotational diffusion times: $10 \mathrm{ps}, 50 \mathrm{ps}$ and $100 \mathrm{ps}$ from top to bottom. 


\section{Page 43 of 43}

1

2

3

4

5

6

7

8

9

10

11

12

13

14

15

16

17

18

19

20

21

22

23

24

25

26

27

28

29

30

31

32

33

34

35

36

37

38

39

40

41

42

43

44

45

46

47

48

49

50

51

52

53

54

55

56

57

58

59

60

Figure 7: Mean square displacement as a function of time for a positive charge on PPV, PPV$m a$ and PPV-da from top to bottom.

Figure 8: Mean square displacement as a function of time for a negative charge on PPV, PPV$m a$ and PPV-da from top to bottom. 